\title{
Análise da pressão arterial domiciliar e no trabalho de auxiliares de enfermagem de um hospital de ensino
}

\author{
Margaret Assad Cavalcante ${ }^{1}$, José Rafael Assad Cavalcante ${ }^{4}$, Maria Nilda Camargo de Barros ${ }^{2}$, Maria \\ Teresa Bombig ${ }^{1}$, Rui Povoa ${ }^{4}$, Francis Silva Lopes ${ }^{3}$, José Rodolfo Assad Cavalcante ${ }^{4}$, Fernando Rodrigues \\ Pimentel Filho \\ ${ }^{1}$ Faculdade de Medicina, ${ }^{2}$ Curso de Enfermagem e ${ }^{3}$ Curso de Fisioterapia da UNOESTE, Presidente Prudente, SP; ${ }^{4}$ Médico \\ Especialista em Cardiologia.
}

\section{Resumo}

O objetivo da pesquisa foi comparar a pressão arterial de profissionais auxiliares de enfermagem em dois momentos distintos durante o trabalho profissional e no momento do descanso do trabalho. Participaram do estudo 31 auxiliares de enfermagem de um hospital de ensino que trabalhavam em regime de 12 horas de trabalho e 36 horas de descanso. Para verificação da pressão arterial foi utilizado o aparelho digital de Medida Residencial da Pressão Arterial (MRPA) de braço devidamente calibrado. Cada profissional recebeu um aparelho de MRPA para utilizá-lo após treinamento, verificando a pressão arterial durante 3 dias de trabalho e 3 dias de descanso em 3 horários pela manhã. Em relação aos sujeitos da pesquisa $80,65 \%$ eram do sexo feminino e $19,35 \%$ masculino, a faixa etária de maior prevalência foi de 31 a 40 anos. Nenhum participante da pesquisa fumava, relatava uso de álcool ou doenças pré-existentes. $\mathrm{Na}$ aplicação do teste t-Student pareado, ao nível de significância de 0,05 não houve evidências de que as médias fossem diferentes. A pressão arterial sistólica (PAS) trabalho foi de 115,55 $\pm 9,66 \mathrm{mmHg}$ e PAS casa de $114,39 \pm 9,10 \mathrm{mmHg}$ e pressão arterial diastólica (PAD) trabalho de 73,97 $\pm 7,50 \mathrm{mmHg}$ e PAD casa de $72,77 \pm 7,88 \mathrm{mmHg}$. Na avaliação da Freqüência cardíaca (FC), a FC trabalho foi de 83,65 $\pm 9,50$ bpm e FC casa de $79,23 \pm 6,92 \mathrm{bpm}(\mathrm{p}<0,05)$. Os dados da pesquisa não evidenciam diferença estatística significante entre a pressão arterial nos momentos de trabalho e em casa porém, observamos um aumento significativo da freqüência cardíaca.

Palavras-chave: monitoramento, pressão arterial, auxiliares de enfermagem.

\section{Analysis of blood pressure at home and at work for nursing assistants to a teaching hospital}

\begin{abstract}
The aim of this study was to compare the professional nursing auxiliaries' blood pressure in two different moments of time. The first measure was during the work and the second during the rest (out of work). Thirty one nursing auxiliaries of a hospital were included in the study. They have worked for 12 hours and rested for 36 hours. It was used a digital equipment of Home Blood Pressure Monitoring (HBPM) to verify the blood pressure. The equipment was properly calibrated. Each professional received equipment of RMBP to use it after training. The person verified the blood pressure during 3 days of work and 3 days of rest in 3 schedules in the morning. The data was analyzed by Student $t$ test, at the significance level of 0.05 . In relation to the people who participated of the research $80.65 \%$ were women and $19.35 \%$ men. The age group of larger prevalence was from 31 to 40 years $(45.12 \%)$. None of the participants of the research used to smoke, to drink alcoholic drinks or to have preexistent diseases. The means of systolic blood pressure (SBP) and diastolic (DBP) of the work were $115.55 \pm 9.66 \mathrm{mmHg}$ and $73.97 \pm 7.50 \mathrm{mmHg}$, respectively, and at home they were $114.39 \pm 9.10 \mathrm{mmHg}$ and $72.77 \pm 7.88 \mathrm{mmHg}$. The heart rate $(\mathrm{HR})$ at work was $83.65 \pm 9.50 \mathrm{bpm}$ and at home $79.23 \pm 6.92 \mathrm{bpm}(p<0,05)$. The data doesn't evidence significant statistical difference among the SBP and DBP in the moments, however it was observed a significant increase of the heart rate during the work period.
\end{abstract}

Keywords: Monitoring, blood pressure, nursing assistants. 


\section{Introdução}

A elevação da pressão arterial representa um fator de risco independente, linear e contínuo para a doença cardiovascular e, elevações a partir de 115/75 mmHg já demonstram aumento nos índices de mortalidade cardiovascular (LEWINGTON et al., 2002).

A Hipertensão Arterial Sistêmica (HAS) não pode ser vista apenas pelo aspecto dos níveis tensionais elevados, pois na verdade a doença existe num contexto sindrômico com alterações hemodinâmicas, tróficas e metabólicas (NOBRE et al., 2000). Sendo assim, a American Society of Hypertension propôs uma nova definição para a hipertensão arterial, ou seja, a hipertensão é uma síndrome cardiovascular, surgindo a partir de complexos e interrelacionados aspectos etiológicos (GOLDFARB, 2005).

Segundo a V Diretrizes Brasileiras de Hipertensão (2006) inquéritos de base populacional realizados em algumas cidades do Brasil mostram prevalência de hipertensão arterial ( $\geq 140 / 90 \mathrm{mmHg}$ ) de $22,3 \%$ a $43,9 \%$. No Brasil, em 2003, as mortes por doença cardiovascular representaram $27,4 \%$ de todos os óbitos. Excluindo-se as mortes violentas e de origem não definida o índice sobe para 37\%. A HAS está envolvida em $40 \%$ das mortes por doença cerebrovascular e em $25 \%$ das mortes por doença coronariana. Em 2005, foram 1.180.184 internações relacionadas com a doença cardiovascular com um custo global de $\mathrm{R} \$$ 1.323.775.008,28 (V DIRETRIZES BRASILEIRAS DE HIPERTENSÃO, 2006). A prevalência da HAS aumenta progressivamente com a idade em ambos os sexos (VASAN et al., 2001), sendo sempre maior na raça negra em qualquer idade (LESSA, 2001).

Há várias maneiras de se identificar essas alterações da PA, como por método direto (intra-arterial) em ambiente hospitalar e indiretos (auscultatório e oscilatório) no consultório, no domicílio e no trabalho (O'BRIEN et al., 2002; IV DIRETRIZ BRASILEIRA DE HIPERTENSÃO ARTERIAL, 2004).

Um método indireto que pode ser empregado para avaliar as alterações pressóricas no trabalho é a Monitorização Residencial da Pressão Arterial (MRPA), que utiliza equipamentos digitais, compactos, validados e com memória para armazenamento dos dados, impressão ou transmissão dos dados a uma central. A maior parte dos aparelhos validados é para medida de braço. Segundo a II Diretriz para o Uso da Monitorização Residencial da Pressão Arterial (2005) é um método de registro da pressão arterial indireto, realizado pelo paciente ou por outra pessoa treinada, durante a vigília. Dentre as principais indicações destacam-se a avaliação da terapêutica anti-hipertensiva e o seguimento do hipertenso do avental branco (BOMBELLI et al., 2005; KIKUIA et al., 2005).

A MRPA apresenta valores de 30 a 40 $\mathrm{mmHg}$ mais baixos do que a medida no consultório (KIKUIA et al., 2005). Diferente da MRPA da prática clínica diária, a MRPA da pesquisa clínica ainda não tem sua padronização definida (MION et al., 2006).

No tratamento da HAS o conhecimento dos fatores de risco associados e o impacto do tratamento nestes fatores são necessários. Assim, apesar de um controle satisfatório da pressão arterial, outros fatores de risco potencialmente maiores podem se sobrepor, não melhorando a situação clínica do paciente. Desta forma, o tratamento atual da HAS não se resume simplesmente à redução dos níveis pressóricos, mas sim da redução do risco cardiovascular global (VASAN et al., 2001; KSHISAGAR et al., 2006).

Idade, fatores sócio-econômicos, consumo de sal, obesidade, sedentarismo e consumo excessivo de álcool são os principais 
fatores relacionados ao aparecimento e a piora da HAS. Estudos recentes evidenciam o efeito do estresse psicoemocional na reatividade cardiovascular e da pressão arterial (UNGER \& PARATI, 2005). A urbanização, os hábitos sociais e a atividade profissional são determinantes maiores (V DIRETRIZES BRASILEIRAS DE HIPERTENSÃO ARTERIAL, 2006).

Há estudos que evidenciam aumento da pressão arterial sistólica em média de $16 \mathrm{mmHg}$ e da pressão arterial diastólica em média de 13 mmHg no trabalho (CLARK et al., 1987). Entre as atividades profissionais, os profissionais da enfermagem constituem um grupo ocupacional pouco estudado no Brasil, apesar de reconhecida como uma atividade penosa, por lidar com situações de sofrimento, dor e morte e pela continuidade e disponibilidade que seu trabalho requer (REVICKI \& MAY 1989; ESTRYN et al., 1990).

Considerando que há poucos estudos que avaliem o ambiente profissional como fator precipitante da HAS, é importante avaliarmos a pressão arterial no momento de trabalho comparando-a com o momento de descanso e identificar, entre os auxiliares de enfermagem a detecção precoce de possíveis hipertensos e analisar a exposição destes profissionais aos principais fatores de risco para o desenvolvimento da doença hipertensiva.

O presente estudo teve como objetivos verificar a variabilidade da pressão arterial e da freqüência cardíaca no trabalho comparando com o descanso do trabalho, em auxiliares de enfermagem utilizando o método Monitorização Residencial da Pressão Arterial (MRPA) e analisar fatores de risco presente para 0 desenvolvimento de elevações tensionais.

\section{Métodos}

Trata-se de estudo descritivo, exploratório que foi realizado em hospital universitário de interior do Estado de São Paulo. O referido hospital apresenta 250 leitos com 1100 funcionários sendo 400 no serviço de enfermagem. Esta pesquisa foi aprovada pela Comissão de Ética do Hospital Universitário e da Universidade do Oeste Paulista, seguindo as orientações do Conselho Nacional de Ética em Pesquisa (CONEP) baseadas na Declaração de Helsinque.

Foram escolhidos aleatoriamente 31 auxiliares de enfermagem do hospital, que concordaram em participar desta pesquisa, e que apresentassem regime de 12 horas de trabalho e 36 horas de descanso, das enfermarias da clínica médica, pediatria, ginecologia e obstetrícia e moléstias infecciosas.

O profissional foi esclarecido e recebeu informações a respeito da sua participação na pesquisa, através da leitura e assinatura do Termo de Consentimento Livre e Esclarecido.

Antes de iniciar as medidas da pressão arterial, foi aplicado um questionário abordando os dados sóciodemográficos e pessoais (sexo, idade, raça, estado civil, grau de instrução, renda familiar, tempo de trabalho na unidade), e sobre fatores de risco para o desenvolvimento ou agravamento da hipertensão arterial (tabagismo, alcoolismo). Foi investigada por meio da anamnese a presença de doenças pré-existentes.

Para a determinação do índice de massa corpórea (IMC=peso/altura ${ }^{2}$ ) avaliou-se o peso e a altura do indivíduo em uma balança mecânica (Welmy). Foram classificados como casos de obesidade aqueles trabalhadores que apresentaram IMC igual ou maior que 30. Considerou-se como sobrepeso o índice entre 25 e $30 \mathrm{Kg} / \mathrm{m}^{2}$ e normopesos os valores inferiores a $25 \mathrm{Kg} / \mathrm{m}^{2}$ (BRAY, 1991).

Com a concordância, cada profissional foi instruído a fazer um treinamento em dia e hora marcados sobre a verificação da pressão arterial e frequência cardíaca com o aparelho digital de 
Monitorização Residencial da Pressão Arterial (MRPA, Microlife BP 3BTO-A) de braço devidamente calibrado e validado, segundo a II Diretriz para o uso da MRPA (2005). Cada profissional recebeu um aparelho de MRPA que usou durante 3 dias de trabalho e 3 dias de descanso do trabalho, sendo registradas 3 medidas por dia em horários predeterminados pela manhã. $A$ freqüência cardíaca foi também monitorada 3 vezes pela manhã pelo aparelho de MRPA, juntamente com a monitorização da pressão arterial.

As verificações com o MRPA ocorreram no braço dominante e os indivíduos foram instruídos a permanecerem 5 minutos sentados em repouso com os pés apoiados, braços relaxados e, antes de realizarem a verificação da pressão arterial e frequência cardíaca.

Os dados numéricos e os categóricos das características sócio-demográficas foram relatados por meio de freqüência (porcentagem) com que ocorreram.

As medidas da pressão arterial $\mathrm{e}$ freqüência cardíaca foram apresentadas como média das três medidas \pm desvio padrão e submetidos ao teste $t$ de Student pareado, com valor de significância de $p<0,05$.

\section{Resultados}

O estudo foi realizado com 31 profissionais, sendo que a faixa etária de 21 e 40 anos, com maior prevalência entre 31 e 40 anos $(45,16 \%$ do total da amostra). Nenhum dos participantes da pesquisa fumava, relatava uso de álcool ou doenças pré-existente.

Em relação ao sexo, houve um predomínio do sexo feminino na amostra, com prevalência de $80,65 \%$ (25 mulheres) e 6 homens. A raça branca teve prevalência de $74,19 \%$ da amostra.

Em relação ao estado civil, os casados representaram $58,06 \%$ da amostra. Quanto ao grau de instrução percebe-se que 1 indivíduo possui nível universitário completo e 7 incompleto.

A maioria da amostra possuía renda familiar média mensal entre 2 e 4 salários mínimos (58,06\% da amostra).

Em relação ao tempo de Trabalho na Unidade, a maioria dos indivíduos trabalhavam entre 2 e 3 anos na Unidade (Tabela 1).

Tabela 1. Freqüência das características da amostra em relação ao Tempo de Trabalho na Unidade.

\begin{tabular}{lcc}
\hline $\begin{array}{l}\text { Tempo Trabalho } \\
\text { (meses) }\end{array}$ & $\mathbf{n}$ & $\%$ \\
\hline Até 1 ano & 7 & $22,58 \%$ \\
Entre 1 e 2 anos & 4 & $12,90 \%$ \\
Entre 2 e 3 anos & 12 & $38,71 \%$ \\
Mais de 3 anos & 8 & $25,81 \%$ \\
\hline Total & 31 & $100,00 \%$ \\
\hline
\end{tabular}

Na tabela 2 está relatada a freqüência do Índice de Massa Corpórea (IMC). Houve predomínio de IMC até 24,99 e o normopeso correspondeu a $41,94 \%$ da amostra.

Tabela 2. Freqüência da classificação da amostra em relação ao Índice de Massa Corpórea (IMC).

\begin{tabular}{lcc}
\hline IMC & $\mathbf{n}$ & $\%$ \\
\hline Até 24,99 & 13 & $41,94 \%$ \\
25,00 a 29,99 & 11 & $35,48 \%$ \\
30,00 a 34,99 & 7 & $22,58 \%$ \\
\hline Total & 31 & $100,00 \%$ \\
\hline
\end{tabular}

A pressão arterial sistólica no trabalho foi 
$115 \pm 9,66 \mathrm{mmHg}$ e em casa foi de 73,97 $\pm 7,50$ $\mathrm{mmHg}$, não apresentando diferenças estatística $(p>0,05)$. Da mesma forma ocorreu com a pressão arterial diastólica, que foi no trabalho foi $73,97 \pm 97,50 \mathrm{mmHg}$ e em casa foi de $72,77 \pm$ $7,88 \mathrm{mmHg}(p>0,05)$.

A frequência cardíaca apresentou-se elevada no trabalho comparando com o momento em casa sendo $83,65 \pm 9,05$ bpm no trabalho e $79,23 \pm 6,92$ bpm em casa $(p<0,05)$. (Tabela 3$)$.

Tabela 3. Média e desvio padrão da pressão arterial sistólica e diastólica (PAS e PAD) e Freqüência Cardíaca (FC).

\begin{tabular}{ccc}
\hline Local & Parâmetro & Média \\
\hline & PAS $(\mathrm{mmHg})$ & $115,55 \pm 9,66$ \\
TRABALHO & PAD $(\mathrm{mmHg})$ & $73,97 \pm 7,50$ \\
& FC $(\mathrm{bpm})$ & $83,65 \pm 9,05$ \\
\hline \multirow{3}{*}{ CASA } & PAS $(\mathrm{mmHg})$ & $114,39 \pm 9,10$ \\
& PAD $(\mathrm{mmHg})$ & $72,77 \pm 7,88$ \\
& FC $(\mathrm{bpm})$ & $79,23 \pm 6,92^{*}$ \\
\hline
\end{tabular}

Teste t-Student pareado, ${ }^{*} \mathrm{p}<0,05$, comparado com FC do trabalho.

\section{Discussão}

Atenção especial tem sido dada aos chamados estresses ocupacionais, os quais podem desencadear doenças cardiovasculares, como a hipertensão arterial sistêmica (VAN EGEREN, 1992). Como o local de trabalho é muitas vezes o fator gerador do estresse, as chances de se desenvolverem doenças aumentam em função do tipo de atividade que o indivíduo executa.

No presente estudo, nenhum dos profissionais apresentava comorbidades, tabagismo ou alcoolismo. O não uso de álcool e tabagismo pode ser relacionado ao nível mais elevado de instrução e a própria profissão da população estudada.

Entre os participantes, verificou-se que $35,48 \%$ encontravam-se com sobrepeso e
$22,58 \%$ estavam obesos, o que é um fator de risco importante para o desenvolvimento de hipertensão arterial e outras alterações metabólicas como o Diabetes Mellitus tipo 2, dislipidemias e a síndrome metabólica (QUIAU et al., 1998).

$\mathrm{Na}$ amostra estudada não houve diferença estatística nos níveis tensionais no trabalho e em casa. A PA em repouso destes profissionais apresentou-se dentro dos valores de normalidade segundo a II Diretriz para o uso da MRPA (2005). Esse resultado de encontrar esse profissional sadio pode ser em decorrência da menor faixa etária desta população, pois a hipertensão é mais relacionada a faixas etárias superiores (GRIM et al., 1995). Outro fator relacionado aos bons níveis tensionais seria o maior grau de instrução. Quanto mais informada, maiores são os cuidados com a saúde (MEIJERS et al.,1989).

Dados diferentes foram encontrados por Aquino et al. (2001), que observaram elevada prevalência $(40,1 \%)$ de hipertensão arterial em 494 auxiliares de enfermagem de um hospital de emergência em Salvador.

Estudos têm demonstrado que as medidas da pressão arterial no trabalho são mais elevadas do que as obtidas em casa (STENBERG et al., 1995; STERGIOU et al., 2004). De acordo com a Organização Mundial de Saúde (World Health Organization and International Society Of Hypertension, 1989) o impacto do estresse mental e de outras manifestações similares sobre 0 sistema cardiovascular deve constituir-se em preocupação prioritária de todas as instituições governamentais de saúde.

Assim, para melhor monitorar as alterações provocadas por fatores como o estresse, novas técnicas têm sido desenvolvidas nos últimos anos objetivando estudar o comportamento da pressão arterial durante as 
diversas atividades diárias como o trabalho e durante o sono (YAROWS et al., 2000).

Entre essas técnicas encontram-se a MRPA, que permite a realização de medidas para avaliar essas variações pressóricas. Um dos aspectos mais relevantes do uso de MRPA em medicina do trabalho refere-se à ênfase que deve ser dada a fatores emocionais que subitamente podem influenciar na pressão arterial e na freqüência cardíaca (II Diretriz para o Uso da Monitorização Residencial da Pressão Arterial, 2005).

As variações da pressão arterial durante o trabalho de turno foram estudadas previamente por meio da Monitorização Ambulatorial da Pressão Arterial (MAPA) por OHIRA et al. (2000). Esses autores observaram que as variações do ritmo cardíaco promoviam modificações pressóricas significativas na MAPA, caracterizadas pela elevação das cifras sistólicas e aumento da variabilidade pressórica em relação ao trabalho diurno, sugerindo que o trabalho de turno poderá exercer influência como um risco adicional para doenças cardiovasculares (OHIRA et al., 2000).

Estas variações da pressão arterial também foram estudadas em médicos residentes em trabalho de turno em emergência e em um dia comum de trabalho, tendo sido também encontrado aumento da pressão arterial durante o trabalho em emergência (FIALHO et al., 2006).

Também foram demonstradas alterações pressóricas sistólicas maiores no trabalho e em casa, em um grupo de trabalhadores de alta exigência no trabalho, que foi definido como a combinação de alta demanda psicológica e baixa decisão de trabalho em comparação com trabalhadores de baixa exigência no trabalho (VAN EGEREN, 1992).

Essas diferenças, pelo menos em parte, podem ser causadas pelo aumento da reatividade cardiovascular e aos fatores comportamentais relacionados ao manejo do estresse durante os períodos da atividade ocupacional, provocando maior variabilidade da pressão arterial e níveis pressóricos mais elevados (BOONE, 1991; YAMASAKI et al., 1998).

No presente estudo, embora a pressão arterial não tenha se alterado no trabalho, a freqüência cardíaca de repouso que é um dos determinantes da pressão arterial (GUYTON \& HALL, 2006), demonstrou-se mais elevada, com significância estatística. Isso pode ser indício que com o passar do tempo poderá ser um dos fatores que poderá levar à elevação pressórica.

A freqüência cardíaca aumentada pode ser uma possibilidade de um aumento de descarga adrenérgica por ativação do sistema nervoso simpático como uma resposta a uma situação de estresse, embora não o suficiente para provocar elevações pressóricas significantes (GUYTON \& HALL, 2006). Esse fato pode ser interpretado como um sinal de alerta para esses profissionais.

Há muitas décadas tem-se empregado a medida casual da pressão arterial para 0 diagnóstico da HAS, que como se sabe apresenta algumas limitações (HAMILTON et al., 1954). Essas limitações em erros na estimativa real da pressão arterial, visto que o sistema circulatório sofre modificações influenciadas por ritmos circadianos e por atividades físicas, psíquicas e ocupacionais (KAPLAN, 2002; JENNINGS et al., 1986).

Mais estudos que avaliem a resposta cardiovascular desses profissionais da área da saúde com outras faixas etárias e a concomitante avaliação do estresse são necessários no ambiente de trabalho.

De qualquer maneira, achados deste trabalho indicam a necessidade da continuidade da conscientização dos profissionais da saúde sobre os fatores de risco para hipertensão arterial e manejo destes para prevenção primária deste 
dano a saúde.

\section{Referências}

Aquino EMMLL, Magalhães LBNC, Araújo MJ, Almeida MCC, Leto JP. Hipertensão arterial em trabalhadoras de enfermagem - padrão de ocorrência, diagnóstico e tratamento. Arquivos Brasileiros de Cardiologia 2001; 76(3):197-202.

Bombelli M, Sega R, Facchetti R, Corrao G, Polo Friz H, Vertemati AM, Sanvito R, Banfi E, Carugo S, Primitz L, Mancia G. Prevalence and clinical significance of a greater ambulatory versus office blood pressure ('reversed white coat' condition) in a general population. Journal of Hypertension 2005;

23(3):513-20.

http://dx.doi.org/10.1097/01.hjh.0000160206.5878 $\underline{1.07}$

Boone JL. Stress and hypertension. Primare Care 1991; 18(3):623-49.

Bray GA. Obesidad. Instituto Internacional de Ciencias de la Vida. Conocimientos Actuales sobre Nutrición. 6ed. Washington: DC: OPS, 1991.

Cordeiro R, Fischer FM, Filho ECL, Filho DCM. Ocupação e hipertensão. Revista de Saúde Pública 1993; 24(5):380-7.

Clark LA, Denby L, Pregibon D, Harshfield GA, Pickering TG, Blank S, Laragh JH. A quantitative analisys of the effects of activity and time of day on the diurnal variations of blood pressure. Journal Chronic Disease 1987; 40(7):671-81. http://dx.doi.org/10.1016/0021-9681(87)90103-2

Egeren, LFV. The Relationship Between Job Strain and Blood Pressure at Work, at Home, and During Sleep. Psychosomatic Medicine 1992; 54:337-43.

Estryn-Behar M, Kaminski M, Peigne E, Bonnet N, Vaichere E, Gozlan C, Azoulay S, Giorgi M.
Stress at work and mental health status among female hospital workers. British Journal of Industrial Medicine 1990; 47:20-8.

Fialho G, Cavichio L, Povoa R, Pimenta J. Effects of 24-h shift work in the emergency room on ambulatory blood pressure monitoring values of medical residents. American Journal of Hypertension 2006; 19, (10):1005-9. http://dx.doi.org/10.1016/i.amihyper.2006.03.007

Goldfarb, B. ASH Panel Proposes New Hypertension Definition. DOC News American Diabetes Association 2005; 2(7): 1.

Grim CE, Henry JP, Myers H. High blood pressure in blacks: salt, slavery, survival, stress and racism. In: Larach JH, Brenner BM. (Ed.) Hypertension, Pathophysiology, Diagnosis and Management. 2ed. New York: Raven Press Ltd., 1995; 12: 171-207.

Guyton, AC, Hall JE. Textbook of medical physiology. 11ed. Philadelphia: Elsevier Inc., 2006.

Hamilton M, Pickering GW, Roberts JA, Sowry GS. The aetiology of essential hypertension. I. The arterial pressure in the general population. Clinical Science 1954; 13(1):11-35.

IV Diretriz Brasileira de Hipertensão Arterial. Arquivos Brasileiros de Cardiologia 2004; 82 (Supl IV):2-22.

IV Diretriz para uso da Monitorização Ambulatorial da Pressão Arterial / II Diretriz para uso da Monitorização Residencial da Pressão Arterial. Arquivos Brasileiros de Cardiologia 2005; 85(Supl II):1-18.

Jennings G, Nelson L, Nestel P, Esler M, Korner $P$, Burton D, Bazelmans J. The effects of changes in physical activity on major cardiovascular risk factors, hemodynamics, sympathetic function and glucose utilization in man: a controlled study of four levels of activities. 
Circulation $\quad$ 1986; 73(1):30-40. http://dx.doi.org/10.1161/01.CIR.73.1.30

Kaplan, NM. Clinical hypertension. 8ed. Philadelphia: Williams \& Wilkins, 2002.

Kikuya M, Ohkubo T, Asayama K, Metoki H, Obara T, Saito S, Hashimoto J, Totsune K, Hoshi H, Satoh H, Imai Y. Ambulatory blood pressure and 10-year risk of cardiovascular and noncardiovascular mortality: the Ohasama study. Hypertension 2005; 45(2):240-5. http://dx.doi.org/10.1161/01.HYP.0000152079.045 $\underline{53.2 c}$

Kshirsagar AV, Carpenter M, Bang H, Wyatt SB, Colindres RE. Blood pressure usually considered normal is associated with an elevated risk of cardiovascular disease. American Journal of Medicine 2006; 119(2):133-41. http://dx.doi.org/10.1016/j.amjmed.2005.08.023

Lessa, I. Epidemiologia Insuficiência Cardíaca e da Hipertensão Arterial Sistêmica no Brasil. Revista Brasileira de Hipertensão 2001; 8(4):38392.

Lewington S, Clarke R, Qizilbash N et al. Agespecific relevance of usual blood pressure to vascular mortality: a meta-analysis of individual data for one million adults in 61 prospective studies. Lancet 2002; 360(9349):1903-13. http://dx.doi.org/10.1016/S0140-6736(02)11911-8

Mion D, Nobre F, Gomes MA. Monitorização Residencial da Pressão Arterial. São Paulo: Manole, 2006.

Nobre F, Lima N, Costa K. Hipertensão Arterial: Conceito, Classificação e Critérios Diagnósticos In: Manual de Cardiologia SOCESP. São Paulo: Atheneu, 2000. p.303-30.

O'Brien E, Pickering T, Asmar R, Myers M, Parati G, Staessen J, Mengden T, Imai Y, Waeber B, Palatini P, Gerin W. Working Group on Blood Pressure Monitoring of The European Society of
Hypertension International Protocol for validation of blood pressure measuring devices in adults. Blood Pressure Monitoring 2002; 7:3-17. http://dx.doi.org/10.1097/00126097-200202000$\underline{00002}$

Ohira T, Tanigawa $\mathrm{T}$, Iso $\mathrm{H}$, Odagiri $\mathrm{Y}$, Takamiya T, Shimomitsu T, Hayano J, Shimamoto T. Effects of shift work on 24-hour ambulatory blood pressure and its variability among Japanese workers. Scandinavian Journal Work, Environment \& Health 2000; 26(5):421-6. http://dx.doi.org/10.5271/sjweh.563

Pickering TG. Recommendations for the use of home (self) and ambulatory blood pressure monitoring. American Journal of Hypertension 1996; 9:1-11. $\quad$ http://dx.doi.org/10.1016/08957061(95)00341-X

Quiau Q, Rajala U, Keinänen-Kiukaanniemi S. Hypertension, hiperinsulinaemia and obesity in middle-aged Finns with impaired glucose tolerance. Journal of Human Hypertension 1998; 12(4):265-9.

http://dx.doi.org/10.1038/sj.jhh.1000602

Revicki DA, May HJ. Organizational characteristics, occupational stress, and mental health in nurses. Behavioral Medicine Spring 1989;

30-6.

http://dx.doi.org/10.1080/08964289.1989.9935149

Sternberg H, Rosenthal T, Shamiss A, Green M. Altered circadian rhythm of blood pressure in shift workers. Journal of Human Hypertension 1995; $9(5): 349-53$.

Stergioun G, Mengden T, Padfield PL, Parati G, O'Brien E. Self monitoring of blood pressure at home. British Medical Journal 2004; 329:870-1. http://dx.doi.org/10.1136/bmi.329.7471.870

Unger T, Parati G. Acute stress and long lasting blood pressure elevation: a possible cause of established hypertension? Journal of 
Hypertension 2005; 23(5):261-3.

http://dx.doi.org/10.1097/00004872-200502000-

$\underline{00004}$

V Diretrizes Brasileiras de Hipertensão Arterial. Sociedade Brasileira de Cardiologia, Sociedade Brasileira de Hipertensão, Sociedade Brasileira de Nefrologia. 2006.

Van Egeren LF. The Relationship Between Job Strain and Blood Pressure at Work at Home, and During Sleep. Psychosomatic Medicine 1992; 54(3):337-43.

Vasan RS, Larson MG, Leip EP, Kannel WB, Levy D. Assessment of frequency of progression to hypertension in non-hypertensive participants in the Framingham Heart Study: a cohort study. Lancet 2001; 358(9294):1682-86. http://dx.doi.org/10.1016/S0140-6736(01)06710-1

Vasan RS, Larson MG, Leip EP, Evans JC, O'Donnell CJ, Kannel WB, Levy D. Impact of highnormal blood pressure on the risk of cardiovascular disease. New England Journal of Medicine 2001; 345(18):1291-7. http://dx.doi.org/10.1056/NEJMoa003417

World Health Organization And International Society Of Hypertension. Mild Hypertension Liaison Committee Memorandum. Guidelines for management of mild hypertension. WHO Bull 1989; 67:493-8.

Yamasaki F, Schwartz JE, Gerber LM, Warren K, Pickering TG. Impact of shift work and race/ethnicity on the diurnal rhythm $\mathrm{BP}$ and catecholamines. Hypertension 1998; 32(3): 41723.

Yarows AS, Julius S, Pickering TG. Home Blood pressure monitoring. Archives of Internal Medicine 2000; 160:1251-7. http://dx.doi.org/10.1001/archinte.160.9.1251 